\title{
Studies of Red Blood Cell Aggregation and Blood Oxygenation on the Basis of the Optoacoustic Effect in Biological Media
}

\author{
Denis A. Kravchuk* and Ksenia A. Voronina** \\ Southern Federal University, 2 Shevchenko str., Building E, Taganrog 347922, Russian Federation \\ *e-mail: kravchukda@sfedu.ru \\ **e-mail: kususha-voronina@yandex.ru
}

\begin{abstract}
The most important task in the field of medical diagnostics is the creation and application of new diagnostic tools and methods, as well as diagnosis of physiological processes. To solve this problem, one may use the optoacoustic (OA) effect - thermooptical excitation of acoustic waves in a liquid medium under laser radiation absorption. This study utilizes numerical methods for modeling processes of oxygen saturation and red blood cells aggregation in the blood. Models of oxygenated red blood cells and their distribution in the blood are constructed using the Monte Carlo method. The acoustic signal from oxygenated and deoxygenated red blood cells was simulated at various levels of oxygen saturation. It was found that the level of spectral power density of the acoustic signal varies significantly and allows one to estimate the percentage of oxygen saturation of red blood cells in the blood with implementation of OA method. A model for the aggregation of red blood cells, combining red blood cells into clusters by hexagonal packing, was developed to increase compactness. It was found that using the OA method, it is possible to determine the level of red blood cells aggregation and of hematocrit level. (C) 2020 Journal of Biomedical Photonics \& Engineering.
\end{abstract}

Keywords: photoacoustic effect; the level of red blood cells aggregation; Monte Carlo method; spherical source.

Paper \#3355 received 26 Feb 2020; revised manuscript received 17 Mar 2020; accepted for publication 21 Mar 2020; published online 27 Mar 2020. doi: 10.18287/JBPE20.06.010307.

\section{Introduction}

In the conditions of development and improvement of medicine, it becomes necessary to develop and study new diagnostic methods, one of which is the optoacoustic (OA) effect. A numerical analysis of the optical characteristics of biological media provides necessary information for effective diagnosis. Modern methods for determining the optical characteristics of biological media require implementation of in vitro analysis, i.e. determination of attenuation by spectrophotometry, fluorescence spectroscopy, Raman spectroscopy, etc. [1]. The advantages of OA method during in vivo analysis reduce the time for deciding on the treatment method. There are numerous possibilities for studying blood composition in vitro, but noninvasive analysis is of great interest as it is a convenient and comfortable method that does not change the morphological composition of the blood [2].

Establishing the level of aggregation of red blood cells (AE) in the blood is important in the diagnosis of blood. Red blood cell aggregation requires clinical diagnosis; AE growth may lead to the development of diseases such as diabetes mellitus, hypertension, anemia, inflammation, etc. [3, 4]. In addition, the increase in aggregation may occur as a result of "side effects" of medications and may lead to blockade of small vessels by aggregates, and difficulties in transporting oxygen in human blood. In this regard, the analysis of aggregation level and oxygen saturation must be carried out for patients undergoing treatment in medical institutions [5-7]. To measure the level of red blood cell aggregation and oxygen saturation in the 
blood, non-invasive techniques, for example utilizing AO effect, are needed. In order to do this, it is necessary to conduct research utilizing mathematical modeling. The authors in Ref. [8] performed mathematical modeling of the formation of OA signal from red blood cells without taking into account a backscattering signal. The authors of Ref. [9] performed optical diagnostics of tissues and their visualization. The authors carried out theoretical and experimental studies of AO signal with laser excitation [10]; they conducted experimental studies of AO effect in model suspensions in the presence of nanotubes and nanofibers without red blood cells modeling.

The aim of the work is the modeling of red blood cells considering oxygen saturation using the Monte Carlo method, development of a mathematical model of red blood cell aggregation by hexagonal packing, and calculation of acoustic signals generated as a result of OA conversion from red blood cells to establish the possibility of aggregation level and oxygen saturation estimation.

\section{Materials and Methods}

A workflow of OA imaging consists in detecting broadband ultrasonic signals generated in biological mediums by laser radiation absorbing. The advantage of the method, in contrast to the methods of radiography and computed tomography, is the application of lowenergy photons and ultrasonic waves.

Ultrasonic wave in biological tissues allows obtaining an improved signal-to-noise ratio and high spatial resolution in deep tissues with high contrast. Spatial resolution is determined by ultrasonic focal power and ultrasonic passband. Biological tissues contain up to $70 \%$ of water, therefore, the distribution of the OA signal will be considered like that in water. When using the OA effect, tissues are irradiated with a laser using short pulses (with a duration of several nanoseconds). The optical and thermoelastic properties of the tissue absorbing laser radiation are studied by recording acoustic pressure using ultrasonic transducers [11]. To enhance the signal, the use of nanosized agents is effective. Carbon nanotubes (up to $100 \mathrm{~nm}$ in size) strongly absorb laser radiation. This allows one to use low-power lasers to prevent skin damage [12-14].

In Refs. [15, 16] (mathematical models have described the formation of an OA signal from an erythrocyte model as a spherical absorption source) the authors concluded that the amplitude of the OA signal monotonically increases with an increase of red blood cells concentration, which makes it possible to determine the hemoglobin level. Thus, it is proposed to use the OA effect to establish the degree of red blood cells aggregation.

To solve the stated problems, it is proposed to use signal processing methods, system analysis, and mathematical statistics. In mathematical modeling, the Matlab R2014b environment has been used as a toolkit.

The geometry of the OA modeling is shown in Fig. 1. The red and blue filled circles are two types of
OA sources with different absorption coefficients, occupying the laser-illuminated region.

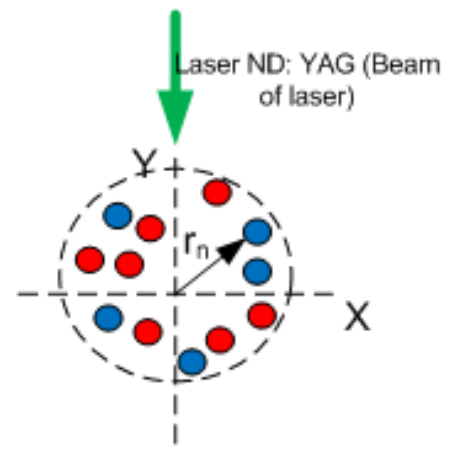

Fig. 1 Two types of acoustic signal sources (red - oxygenated red blood cells, and blue circles deoxygenated red blood cells).

The analytical expression of the acoustic field formed by the liquid sphere may be written in the form of Eq. (1) [16]:

$$
p_{f}^{1}(q)=\frac{i \mu \beta I_{0} v_{\mathrm{s}} \alpha^{2}}{C_{p} r} \frac{[\sin q-q \cos q] e^{i k_{f}(r-\alpha)}}{q^{2}\left[(1-q)\left(\frac{\sin q}{q}\right)-\cos q+i \rho \nu \sin q\right]},
$$

where $\mu$ is the absorption coefficient, $C_{P}$ is isobaric specific heat, $\beta$ is the coefficient of thermal expansion, $\alpha$ is the radius of the absorbing sphere, $\rho=\rho_{\mathrm{s}} / \rho_{\mathrm{f}}$ and $v=v_{\mathrm{s}} / v_{f}$ are the dimensionless density and speed of sound $\left(v_{\mathrm{s}}\right.$ is the speed of sound in red blood cells, $v_{f}$ - the speed of sound in blood plasma), respectively.

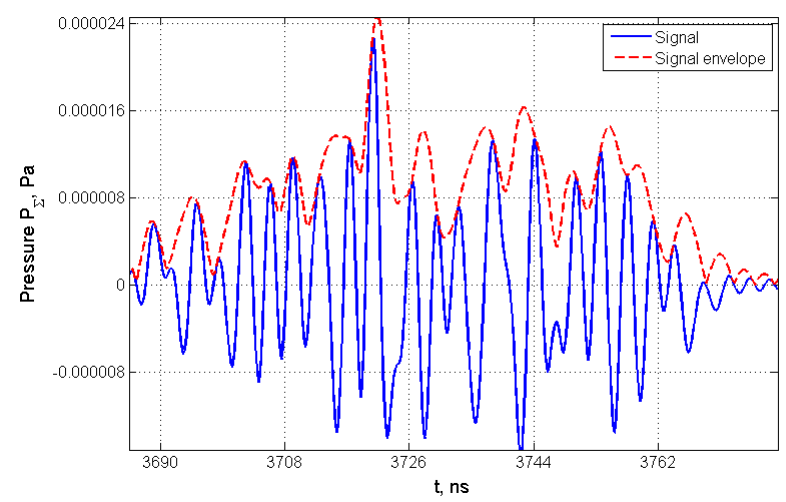

Fig. 2 Acoustic signal at $99 \%$ oxygen saturation (with signal envelope).

Fig. 2 shows the pressure curve of the acoustic signal for a simulated sample with saturation $\left(\mathrm{SO}_{2}=100 \%\right)$ while exposition with $1064 \mathrm{~nm}$ laser.

Mathematical modeling of oxygenated and deoxygenated erythrocytes consists in the formation of a three-dimensional realization of tissues with the condition of non-overlapping spheres representing simulated red blood cells in a volume of $100 \times 100 \times 100 \mathrm{\mu m}^{3}$. The simulated volume is filled with 
red blood cells approximated in the form of liquid spheres with a hematocrit level of $40 \%$ since it is close to normal for human blood (Fig. 3). Moreover, in the model, oxygenated and deoxygenated red blood cells have certain densities, sound velocities, and absorption coefficients. Similarly, we form tissues with aggregated red blood cells (Fig. 4). In this case, we find the spatial positions of randomly distributed red blood cells within the study area of $100 \times 100 \mu \mathrm{m}^{2}$. The number of clusters also determines the number of cells that could be attached to one cluster. The maximum packing density of red blood cells was achieved using hexagonal packing for each cluster $[15,18-20]$.

\section{RESULTS}

The model allows one the formation of red blood cells into aggregates with varying degrees of filling Fig. 4.

Fig. 3 shows the spectral power densities of the acoustic signal at $1 \%, 25 \%, 50 \%$, and $100 \%$ of saturation. A large range of simulated levels of saturation is given in order to show the possibilities of the OA method for recording oxygen saturation. As the oxygen saturation of $\mathrm{SO}_{2}$ increased, the spectral power of the signal also increased. An algorithm has been developed for modeling the implementation of 2D tissues, consisting of oxygenated red blood cells and deoxygenated red blood cells [15].
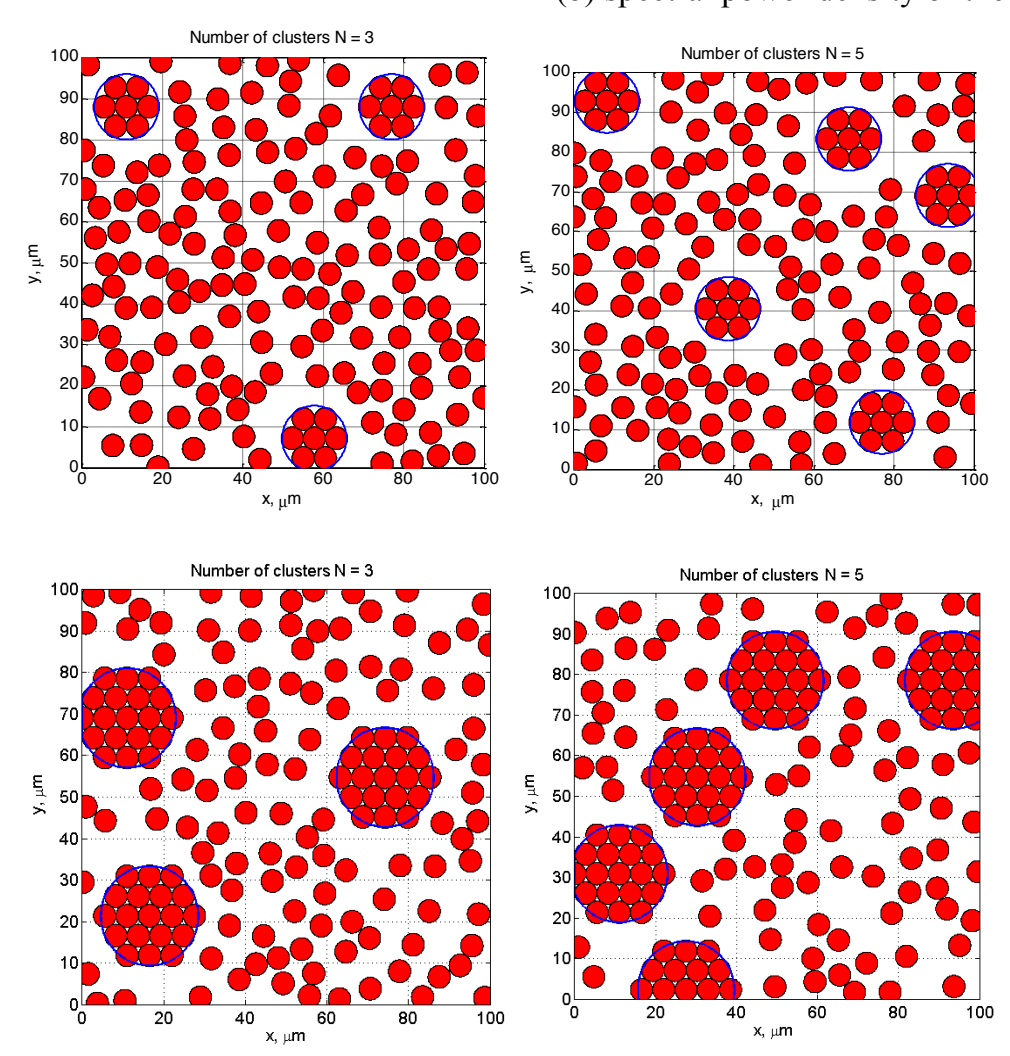

Fig. 4 Modeling of various levels of red blood cell aggregation in the blood. 


\section{Discussion}

In Ref. [10] the authors carried out experimental measurements of an acoustic signal with OA effect in liquid solutions with carbon nanotubes. The aim of the study was to demonstrate a possibility to control of delivering nano-drugs to the required point in the body. For this purpose, an experimental setup and model solutions were developed. The authors of the study [23] calculated the profiles of OA signals and reconstructed the absorption coefficients for different wavelengths of laser irradiation. The study of biological fluids was carried out in reflection mode for OA microscopy.

The studies conducted as part of our article allow us to pre-model acoustic signals with the OA effect in various solutions, which facilitates preparation for experiments and allows us to correctly interpret the results. The obtained theoretical studies will be verified during the experiments using the LIMO 100-532/1064U setup (Fig. 6) [21, 22]. The simulation results and subsequent experimental measurements complements to the theoretical studies carried out in Refs. [8, 11]. OA signal recording will be performed using the NI PXI 1042Q modular measuring complex, for which a block diagram of a "virtual" instrument based on LabVIEW has been developed. It is difficult to carry out experimental measurements with natural blood for several reasons, such as the difficulty of obtaining native blood, application of drugs that slow down coagulation, which will affect the measurement results. The simulated erythrocytes will be made of polymer microspheres, disks which were manufactured by Diapharm LLC (St. Petersburg) corresponding to shapes and sizes of erythrocytes, with an optical absorption coefficient close to that of erythrocytes in equivalent concentrations.

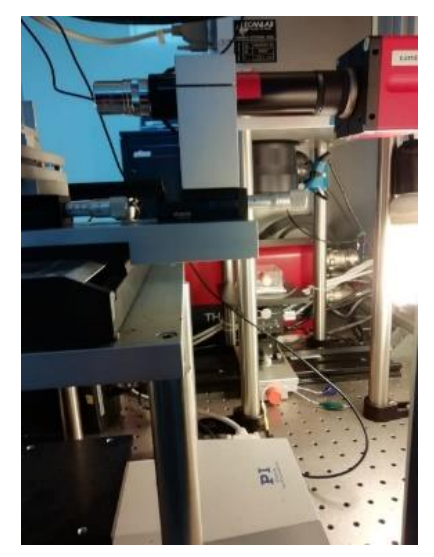

Fig. 6 Experimental setup LIMO 100-532/1064-U.

\section{Conclusion}

The proposed model was used to study the change in the amplitude of the acoustic signal and the spectral power density of the signal generated as a result of the OA effect, depending on the level of red blood cells aggregation and oxygen saturation. A mathematical model of red blood cell aggregation by hexagonal packing has been developed. The signals generated as a result of the OA effect from red blood cells were calculated. The possibility of recording the level of aggregation and oxygen saturation using the OA method is shown.

\section{Disclosures}

All authors declare that there is no conflict of interests in this paper.

\section{References}

1. B. G. Andryukov, A. A. Karpenko, E. V. Matosova, and I. N. Lyapun, "Raman spectroscopy as a modern diagnostic technology for study and indication of infectious agents (review)," Sovremennye Tehnologii v Medicine 11(4), 161 (2019) [in Russian].

2. M. Rui, W. Bost, E. C. Weiss, R. Lemor, and M. C. Kolios, "Photoacoustic microscopy and spectroscopy of individual red blood cells," Proceeding of Biomedical Optics and 3-D Imaging, BSuD93 (2010).

3. H. J. Meiselman, "Red blood cell aggregation: 45 Years being curious,” Biorheology 46(1), 1-19 (2009).

4. J. J. Bishop, A. S. Popel, M. Intaglietta, and P. C. Johnson, "Rheological effects of red blood cell aggregation in the venous network: a review of recent studies," Biorheology IOS Press 38(2-3), 263-274 (2001).

5. S. Kim, A. S. Popel, M. Intaglietta, and P. C. Johnson, "Aggregate formation of erythrocytes in postcapillary venules," merican Journal of Physiology-Heart and Circulatory Physiology 288(2), H584-H590 (2005).

6. H. Bäumler, B. Neu, E. Donath, and H. Kiesewetter, "Basic phenomena of red blood cell rouleaux formation," Biorheology 36(5-6), 439-442 (1999).

7. A. Chabanel, M. H. Horellou, J. Conard, and M. M. Samama, "Red blood cell aggregability in patients with a history of leg vein thrombosis: Influence of post-thrombotic treatment," British Journal of Haematology 88(1), 174-179 (1994).

8. R. K. Saha, M. C. Kolios, "A simulation study on photoacoustic signals from red blood cells," The Journal of the Acoustical Society of America 129(5), 2935-2943 (2011).

9. V. V. Tuchin (Ed.), Handbook of Optical Biomedical Diagnostics, SPIE Press, PM107, Bellingham (2002).

10. G. Y. Dzhuplina, I. B. Starchenko, "System and methodology for the study of nanoscale artifacts in the bloodstream," Izvestiya SFedU. Engineering Sciences 8(10), 61-64 (2010) [in Russian]. 
11. I. V. Mitrofanova, I. V. Milto, I. V. Sukhodolo, and G. Y. Vasyukov, "Possibilities of biomedical application of carbon nanotubes," Byulleten' sibirskoy meditsiny 13(1), 135-144 (2014) [in Russian].

12. N. V. Sayapina, A. A. Sergievich, T. A. Batalova, M. A. Novikov, A. N. Asadcheva, V. V. Chaika, and K. S. Golokhvast, "Ecological and toxicological hazards of carbon nanotubes: a review of Russian publications," Izvestiya of the Samara Russian Academy of Sciences scientific center 16(5), 949-953 (2014) [in Russian].

13. K. A. Voronina, D. A. Kravchuk, I. B. Starchenko, D. V. and Orda-Zhigulina, "The method of express blood analysis using contrast agents - nanoparticles," In the collection: Nanotekhnologii: obrazovaniye, nauka, innovatsii, Sbornik statey X Vserossiyskoy nauchno-prakticheskoy konferentsii, 59-61 (2019) [in Russian].

14. D. A. Kravchuk, I. B. Starchenko, "Model assessment of the level of red blood cell aggregation by the optoacoustic method," Caspian Journal: Control and High Technologies 4(40), 174-183 (2017) [in Russian].

15. D. A. Kravchuk, I. B. Starchenko, "Mathematical modeling of the optoacoustic signal from red blood cells," Journal of New Medical Technologies 25(1), 96-101 (2018) [in Russian].

16. H. M. Lai, K. Young, "Theory of the pulsed optoacoustic technique," The Journal of the Acoustical Society of America 72(6), 2000-2007 (1982).

17. D. A. Kravchuk, "A mathematical model for detecting intra-erythrocyte pathologies using the optoacoustic method," Biomedical Photonics 7(3), 36-42 (2018).

18. D. A. Kravchuk, I. B. Starchenko, "Application of optoacoustic method for model calculation of non-aggregated erythrocytes and determination of hematocrit level," In the collection: Proceedings of the 2018 international conference on "Physics, mechanics of new materials and their applications", 307-316 (2019).

19. D. A. Kravchuk, I. B. Starchenko, "A theoretical model for diagnosing the effect of oxygen saturation of red blood cells using optoacoustic signals," Applied Physics 4, 89-94 (2018) [in Russian].

20. D. A. Kravchuk, "Simulation of acoustic signals with an optoacoustic effect for the detection of red blood cells of various shapes by a laser cytometer," Applied Physics 5, 93-99 (2019).

21. D. A. Kravchuk, "Experimental studies on the excitation and registration of an optoacoustic signal in a liquid," IEEE International Siberian Conference on Control and Communications (SIBCON-2019), 8729639 (2019).

22. I. B. Starchenko, D. A. Kravchuk, and I. A. Kirichenko, "An Optoacoustic Laser Cytometer Prototype," Biomedical Engineering 51(5), 308-312 (2018).

23 P. S. Grashin, A. A. Karabutov, A. A. Oraevsky, I. M. Pelivanov, and E. V. Savateeva, "Distribution of the intensity of laser radiation in strongly scattering media: Monte Carlo simulation, theoretical analysis and results of optical-acoustic measurements," Quantum Electronics 32(10), 868-874 (2002). 